\title{
Coalescence overgrowth of GaN nanocolumns on sapphire with patterned metal organic vapor phase epitaxy
}

\author{
Tsung-Yi Tang, ${ }^{1}$ Wen-Yu Shiao, ${ }^{1}$ Cheng-Hung Lin, ${ }^{1}$ Kun-Ching Shen, ${ }^{1}$ Jeng-Jie Huang, \\ Shao-Ying Ting ${ }^{1}{ }^{1}$ Tzu-Chi Liu, ${ }^{1}$ C. C. Yang, ${ }^{1, a)}$ Chiu-Lin Yao, ${ }^{2}$ Jui-Hung Yeh, ${ }^{2}$ \\ Ta-Cheng Hsu, ${ }^{2}$ Wei-Chao Chen, ${ }^{3}$ Hsu-Cheng Hsu, ${ }^{3}$ and Li-Chyong Chen ${ }^{3}$ \\ ${ }^{1}$ Institute of Photonics and Optoelectronics and Department of Electrical Engineering, National Taiwan \\ University, 1, Roosevelt Road, Section 4, Taipei 10617, Taiwan, Republic of China \\ ${ }^{2}$ Epistar Corporation, Hsinchu 30078, Taiwan, Republic of China \\ ${ }^{3}$ Center for Condensed Matter Science, National Taiwan University, Taipei 10617, Taiwan, \\ Republic of China
}

(Received 20 September 2008; accepted 25 November 2008; published online 16 January 2009)

\begin{abstract}
High-quality coalescence overgrowth of patterned-grown GaN nanocolumns on $c$-plane sapphire substrate with metal organic chemical vapor deposition is demonstrated. Although domain structures of a tens of micron scale in the overgrown layer can be identified with cathodoluminescence measurement, from atomic force microscopy (AFM) measurement, the surface roughness of the overgrown layer in an area of $5 \times 5 \mu \mathrm{m}^{2}$ is as small as $0.411 \mathrm{~nm}$, which is only one-half that of the high-quality GaN thin-film template directly grown on sapphire substrate (the control sample). Based on the AFM and depth-dependent x-ray diffraction measurements near the surface of the overgrown layer, the dislocation density is reduced to the order of $10^{7} \mathrm{~cm}^{-2}$, which is one order of magnitude lower than that of the control sample and two to three orders of magnitude lower than those of ordinary $\mathrm{GaN}$ templates for fabricating light-emitting diodes. Also, the lateral domain size, reaching a level of $\sim 2.7 \mu \mathrm{m}$, becomes three times larger than the control sample. Meanwhile, the ratio of photoluminescence intensity at room temperature over that at low temperature of the overgrown sample is at least six times higher than that of the control sample. Although the strain in nanocolumns is almost completely released, a stress of $\sim 0.66 \mathrm{GPa}$ is rebuilt when the coalescence overgrowth is implemented. (C) 2009 American Institute of Physics. [DOI: 10.1063/1.3065527]
\end{abstract}

\section{INTRODUCTION}

The growth of GaN nanocolumns on $c$-plane sapphire or $\mathrm{Si}$ substrate is appealing because the nanocolumns can be dislocation-free due to the lateral strain relaxation in the column geometry. ${ }^{1-5}$ Also, an InGaN/GaN light-emitting diode (LED) has been grown on nanocolumns to achieve high indium content and high-crystal quality. ${ }^{6}$ However, for device fabrication, a planar geometry is preferred. Therefore, coalescence overgrowth on such high-crystal-quality GaN nanocolumns becomes an important issue. With coalescence overgrowth, we can prepare low-dislocation-density $\mathrm{GaN}$ templates for device fabrication. GaN nanocolumns can be grown by molecular-beam epitaxy (MBE) and metal organic chemical vapor deposition (MOCVD) with the methods of self-organized growth, ${ }^{7,8}$ regrowth on a selective mask, ${ }^{9}$ and catalyst-assisted growth. ${ }^{10,11}$ To implement parallel and vertical nanocolumns with MOCVD, normally patterned growth is preferred. Regularly arranged $\mathrm{GaN}$ nanocolumns created via patterned MOCVD growth by using interferometric lithography have been demonstrated. ${ }^{12}$ Nanocolumn growth followed by coalescence overgrowth with MBE has also been reported. ${ }^{13}$ Recently, MOCVD coalescence overgrowth of MBE-grown self-organized GaN nanocolumns on Si substrate was reported by this group. ${ }^{14}$ However, further im-

\footnotetext{
${ }^{a)}$ Tel.: 886-2-23657624. FAX: 886-2-23652637. Electronic mail: ccy@cc.ee.ntu.edu.tw.
}

provement of the quality of the overgrown layer is needed. The quality of the overgrown layer depends on the quality of column array, including its regularity.

In this paper, high-quality coalescence overgrowth of patterned-grown $\mathrm{GaN}$ nanocolumns with MOCVD on $c$-plane sapphire substrate is demonstrated. A fairly regular pattern of nanocolumn based on nanoimprint lithography is first deposited, followed by a two-dimensional growth procedure, leading to a smooth overgrown layer of significantly higher crystal and optical qualities. Two depth-dependent $\mathrm{x}$-ray diffraction (XRD) techniques are used to monitor the progress of crystal quality during the process of coalescence overgrowth. The XRD and photoluminescence (PL) comparisons between the overgrown sample and a GaN thin-film template used for nanocolumn growth show the significantly superior quality near the surface of the overgrown layer. Such a GaN template is useful not only for high-quality device fabrication but also for an easier laser lift-off process in fabricating vertical LEDs. In Sec. II, the nanocolumn growth and coalescence overgrowth conditions are discussed. Also, the characterization techniques are presented. The characterization results are shown in Sec. III. Finally, conclusions are drawn in Sec. IV.

\section{GROWTH CONDITIONS AND CHARACTERIZATION TECHNIQUES}

To first prepare the pattern for nanocolumn growth on a $\mathrm{GaN}$ template, which consists of a $2 \mu \mathrm{m} \mathrm{GaN}$ thin film 
grown at $1050{ }^{\circ} \mathrm{C}$ after the nucleation layer of $40 \mathrm{~nm}$ grown at $530{ }^{\circ} \mathrm{C}$ on $c$-plane sapphire substrate, an $80 \mathrm{~nm} \mathrm{SiO}$ layer was deposited at $300{ }^{\circ} \mathrm{C}$ with plasma-enhanced chemical vapor deposition. Then, nanoimprint lithography was applied to open circular holes of $250 \mathrm{~nm}$ in diameter and $500 \mathrm{~nm}$ in distance between the nearest neighboring hole centers with the hexagonal pattern on the $\mathrm{SiO}_{2}$ layer created through reactive ion etching. At the beginning of MOCVD growth, the substrate temperature was set at $1050{ }^{\circ} \mathrm{C}$ with a chamber pressure of 100 torr and a V/III ratio of $1100.5 \mathrm{~s}$ after the growth was started, the flow rates of trimethylgallium (TMGa) and ammonia were alternatively on/off modulated for nanocolumn growth. ${ }^{12}$ During the growth with flow-rate modulation, the flow rates of TMGa and ammonia were $12.5 \mu \mathrm{mol} / \mathrm{min}$ and $500 \mathrm{SCCM}$ (SCCM denotes cubic centimeter per minute at STP), respectively. The durations of supplying TMGa and ammonia were 20 and $30 \mathrm{~s}$, respectively. GaN nanocolumns of $\sim 1 \mu \mathrm{m}$ in height were obtained after a period of $30 \mathrm{~min}$. For coalescence overgrowth, the chamber pressure and V/III ratio were changed into 200 torr and 3900, respectively, with the growth temperature kept at $1050{ }^{\circ} \mathrm{C}$. The continuous flow rates of TMGa and ammonia were $3.5 \mu \mathrm{mol} / \mathrm{min}$ and $1500 \mathrm{SCCM}$, respectively. Under such growth conditions, the growth rate was $\sim 1.3 \mu \mathrm{m} / \mathrm{h}$. Coalescence overgrowth of $90 \mathrm{~min}$ led to an overgrown layer of $\sim 2 \mu \mathrm{m}$ in thickness. To demonstrate the improved quality of the coalescence overgrowth on nanocolumns, a sample of the aforementioned $\mathrm{GaN}$ template for nanoimprint process was used as the control sample for comparison.

The control and overgrown samples were examined with PL and depth-dependent XRD measurements. The PL measurement was excited with a $325 \mathrm{~nm} \mathrm{HeCd}$ laser. In the XRD measurement, two depth-dependent techniques were used for calibrating crystal parameters. First, we used a three-beam XRD technique for plotting the rocking curves in the (01$13) /(0-11-2)$ plane at various depths by changing the x-ray incident angle. ${ }^{15-18}$ In such a measurement, depending on the incident angle of the x-ray beam, the XRD data mainly come from a certain depth of the sample. In the second depthdependent XRD method, a two-beam technique is used for calibrating the screw dislocation density, edge dislocation density, and the lateral domain size based on the equation ${ }^{19}$

$$
\Gamma_{h k l}^{n}=\left(\Gamma_{\mathrm{tilt}} \cos \varphi\right)^{n}+\left(\Gamma_{\mathrm{twist}} \sin \varphi\right)^{n}+(2 \pi / L)^{n} / K_{h k l}^{n} .
$$

Here, $\Gamma_{h k l}$ is the measured full width at half maximum (FWHM) of the diffraction pattern in the $(h k l)$ plane, $\varphi$ is the angle between the diffraction plane and the sample surface, $\Gamma_{\text {tilt }}$ and $\Gamma_{\text {twist }}$ are the FWHMs of the tilt and twist angle distributions, respectively, $L$ is the lateral domain size, and $K_{h k l}$ is the diffraction wavenumber in the $(h k l)$ plane. In Eq. (1), $n$ is a parameter describing the shape of the measured rocking curve with $n=2$ for a Gaussian distribution, $1<n$ $<2$ for a pseudo-Voigt distribution, and $n=1$ for a Lorentzian distribution. The unknowns $L, \Gamma_{\text {tilt }}$, and $\Gamma_{\text {twist }}$ can be obtained by numerically solving a set of three equations of three diffraction planes, i.e., three $(h k l)$ sets. After $\Gamma_{\text {tilt }}$ and $\Gamma_{\text {twist }}$ are obtained, the screw dislocation density, $\rho_{\text {screw }}$, and edge dislocation density, $\rho_{\text {edge }}$, can be obtained through

$$
\begin{aligned}
& \rho_{\text {screw }}=\Gamma_{\text {tilt }}^{2} / 4.36 b_{s}^{2}, \\
& \rho_{\text {edge }}=\Gamma_{\text {twist }}^{2} / 4.36 b_{e}^{2} .
\end{aligned}
$$

Here, $b_{s}\left(b_{e}\right)$ is the length of Burgers vector of the screwtype (edge-type) threading dislocation. For depth discrimination, we use the expression of the probing depth, $t$, as ${ }^{18}$

$$
t=\frac{1}{u}\left(\frac{1}{\sin \omega_{\mathrm{in}}}+\frac{1}{\sin \omega_{\mathrm{ex}}}\right)^{-1} \cos \chi .
$$

Here, $u$ is the decay constant of $\mathrm{x}$-ray intensity in the sample. Also,

$$
\begin{aligned}
& \omega_{\text {in }}=\theta_{B}-\varphi \sin \phi, \\
& \omega_{\text {ex }}=\theta_{B}-\varphi \sin \phi,
\end{aligned}
$$

and

$$
\chi=\varphi \cos \phi .
$$

In Eqs. (5) and (6), $\theta_{B}$ is the Bragg angle of crystal diffraction and $\phi$ is the azimuthal angle of the diffraction vector. By varying the $\phi$ angle, we can probe the crystal quality at different depths.

In our calibration, we consider the three diffraction planes as $(h k l)=(102),(103)$, and (203) and the following parameter values: $u=0.029 \mu \mathrm{m}^{-1}, \quad b_{s} \quad\left(b_{e}\right)$ $=0.5185(0.3189) \mathrm{nm}$, the x-ray wavelength $\lambda=0.154 \mathrm{~nm}$, and $n=1.45-1.52$. For the (102) plane, $\theta_{B}, \varphi$, and $K_{h k l}$ $\left(=4 \pi \sin \theta_{B} / \lambda\right)$ are $24.04^{\circ}, 43.19^{\circ}$, and $33.22 \mathrm{~nm}^{-1}$, respectively. For the (103) plane, $\theta_{B}, \varphi$, and $K_{h k l}$ are $31.71^{\circ}, 32.04^{\circ}$, and $42.87 \mathrm{~nm}^{-1}$, respectively. For the (203) plane, $\theta_{B}, \varphi$, and $K_{h k l}$ are $45.54^{\circ}, 51.38^{\circ}$, and $58.21 \mathrm{~nm}^{-1}$, respectively. Based on the aforementioned measurements and calculations, we can calibrate reasonable values of screw dislocation density, edge dislocation density, and lateral domain size at various sample depths.

\section{CHARACTERIZATION RESULTS OF THE GROWN SAMPLES}

Figure 1(a) shows a tilted plan-view scanning electron microscopy (SEM) image of the hexagonally arranged GaN nanocolumns, which have the cross-sectional shape of hexagon. Here, one can see that with a few exceptions, the sizes of columns are quite uniform. The column heights are about $1 \mu \mathrm{m}$ and the average cross-sectional size is around $300 \mathrm{~nm}$. The column spacing is fixed at $500 \mathrm{~nm}$ by the nanoimprint mold. Along the $c$-axis, the column cross section is also quite uniform. In Fig. 1(b), we show a cross-sectional SEM image demonstrating the bottom of a nanocolumn. Here, the bottom edges of the $\mathrm{SiO}_{2}$ masks (80 nm in thickness) of slanted walls define the $250 \mathrm{~nm}$ hole diameter. The column crosssectional size $(300 \mathrm{~nm})$ is larger than the diameter of the hole. Figures 2(a) and 2(b) show the plan-view and crosssectional SEM images, respectively, of the overgrowth sample. One can see that the overgrown surface is quite smooth. From the cross-sectional image, one can identify three layers, corresponding to the overgrown layer (I), the nanocolumn layer (II), and the GaN template layer (III). We can then estimate the thicknesses of the overgrown layer and 

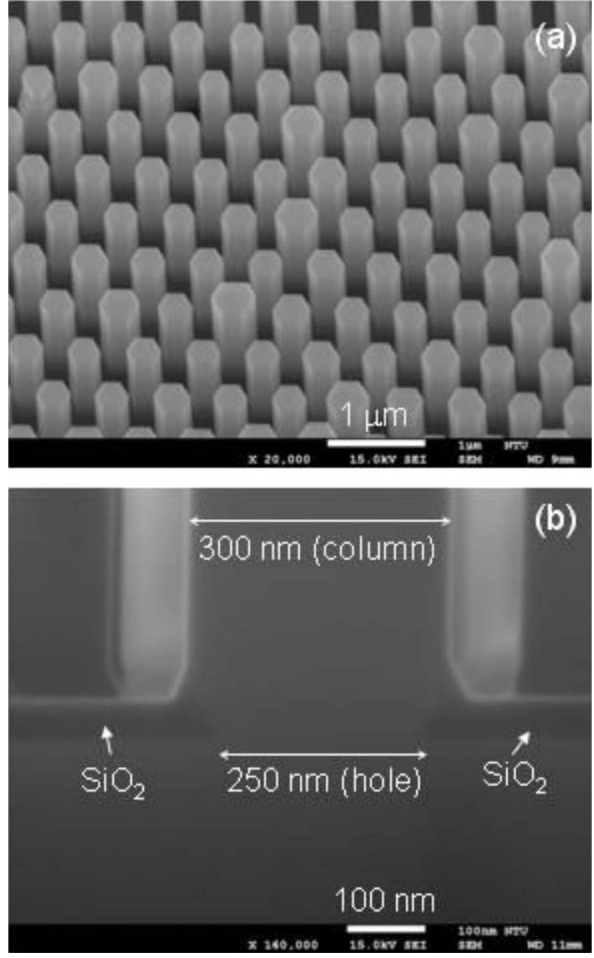

FIG. 1. (a) A tilted plan-view SEM image of hexagonally arranged nanocolumns, which have the cross-sectional shape of hexagon. Except a few relatively larger columns, the sizes and heights of those columns are quite uniform. (b) A cross-sectional SEM image showing the bottom of a nanocolumn. The bottom edges of the $\mathrm{SiO}_{2}$ masks (100 nm in thickness) of slanted walls define the $250 \mathrm{~nm}$ hole diameter. The column cross-sectional size is around $300 \mathrm{~nm}$.

the nanocolumn layer in this overgrown sample to give $\sim 2$ and $\sim 1 \mu \mathrm{m}$, respectively. In preparing the cross-sectional SEM sample, the parts of certain columns buried in the $\mathrm{GaN}$ template were removed, leaving the holes in the GaN template layer. A cross-sectional SEM image demonstrating the interface between the template and nanocolumn layers and the $\mathrm{SiO}_{2}$ masks in between is shown in Fig. 2(c). The air gaps between nanocolumns are partially filled during the overgrowth process. Here, the original column walls are depicted by the vertical dashed lines. The surface roughness of the overgrown layer was monitored with atomic force microscopy (AFM) measurement. In Figs. 3(a) and 3(b), we compare the AFM images $\left(5 \times 5 \mu \mathrm{m}^{2}\right)$ of the control (a) and overgrowth (b) samples. Here, one can clearly see the smoother surface of the overgrowth sample. From the AFM image of $5 \times 5 \mu \mathrm{m}^{2}$ in dimension, we can calibrate the pit density of $3 \times 10^{8} \mathrm{~cm}^{-2}$ and surface roughness of $0.834 \mathrm{~nm}$ in the control sample and the pit density of $2 \times 10^{7} \mathrm{~cm}^{-2}$ and surface roughness of $0.411 \mathrm{~nm}$ in the overgrowth sample. The surface roughness of a $10 \times 10 \mu \mathrm{m}^{2}$ area was calculated to give a slightly larger value at $0.540 \mathrm{~nm}$.

Figures 4(a) and 4(b) show the cross-sectional cathodoluminescence (CL) image of the overgrowth sample with an electron acceleration voltage of $15 \mathrm{kV}$ and the SEM image taken at the same location, respectively. Here, one can see that the emission intensity in the nanocolumn layer is stronger than that in the overgrown layer, manifesting the higher optical quality of the grown nanocolumns. The higher emis-
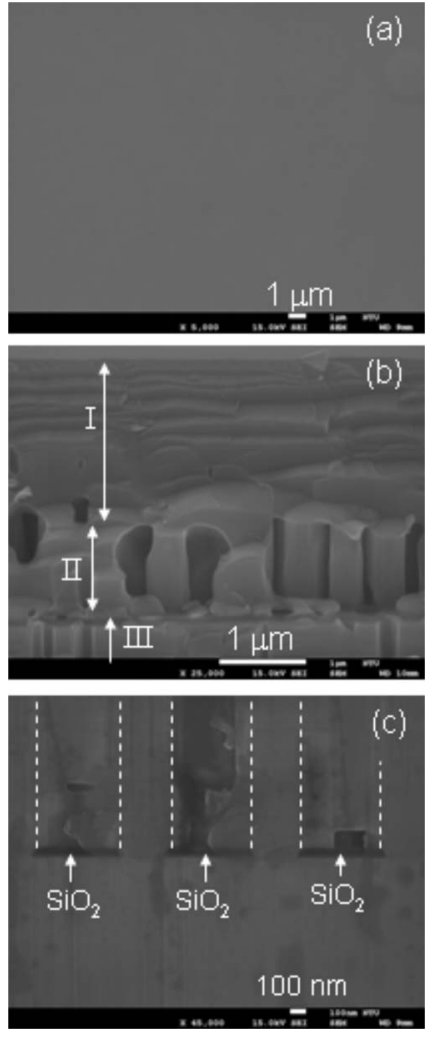

FIG. 2. Plan-view (a) and cross-sectional (b) SEM images of the overgrowth sample. The layers of overgrowth, nanocolumn, and GaN template are indicated with arrows and labeled by I, II, and III, respectively. A cross-sectional SEM image demonstrating the interface between the template and nanocolumn layers and the $\mathrm{SiO}_{2}$ masks in between is shown in part (c). The air gaps between nanocolumns are partially filled during the overgrowth process. The original column walls are depicted by the vertical dashed lines.

sion efficiency of the nanocolumns is attributed to their lower dislocation density ${ }^{12,14}$ that can be due to the dislocation bending behavior when a nanocolumn emerges from a hole on the $\mathrm{SiO}_{2}$ mask during the early stage of nanocolumn growth. ${ }^{20}$ Figures 5(a) and 5(b) show the plan-view CL images of 5000-time magnification with electron acceleration voltages of 6 and $15 \mathrm{kV}$, respectively, at about the same location. With 6 and $15 \mathrm{kV}$ electron acceleration voltages, the penetration depths of the excitation electron are estimated to be 300 and $1300 \mathrm{~nm}$, respectively, in $\mathrm{GaN} .{ }^{21}$ Here, the voids and defects of nearly random distributions result in the random pattern of emission. In a shallower layer, the coalescence process is more complete and hence the dark spot area becomes smaller, as shown in Fig. 5(a). To demonstrate a larger-area distribution, the 1000-time cross-sectional $\mathrm{CL}$ images of 3 and $15 \mathrm{kV}$ in electron acceleration voltage at about the same location are shown in Figs. 6(a) and 6(b), respectively. With the voltage at $3 \mathrm{kV}$, the electron penetration depth is around $90 \mathrm{~nm} .{ }^{21}$ For reference, the plan-view SEM image of the same location is also shown in Fig. 6(c). Here, one can see the domain structure in the overgrown layer with the domain size in the range of several tens of microns. The contrast between the domain centers and boundaries becomes stronger in the CL image of deeper electron penetration. However, the domain patterns are the same at different depths. In other words, the domain pattern was 

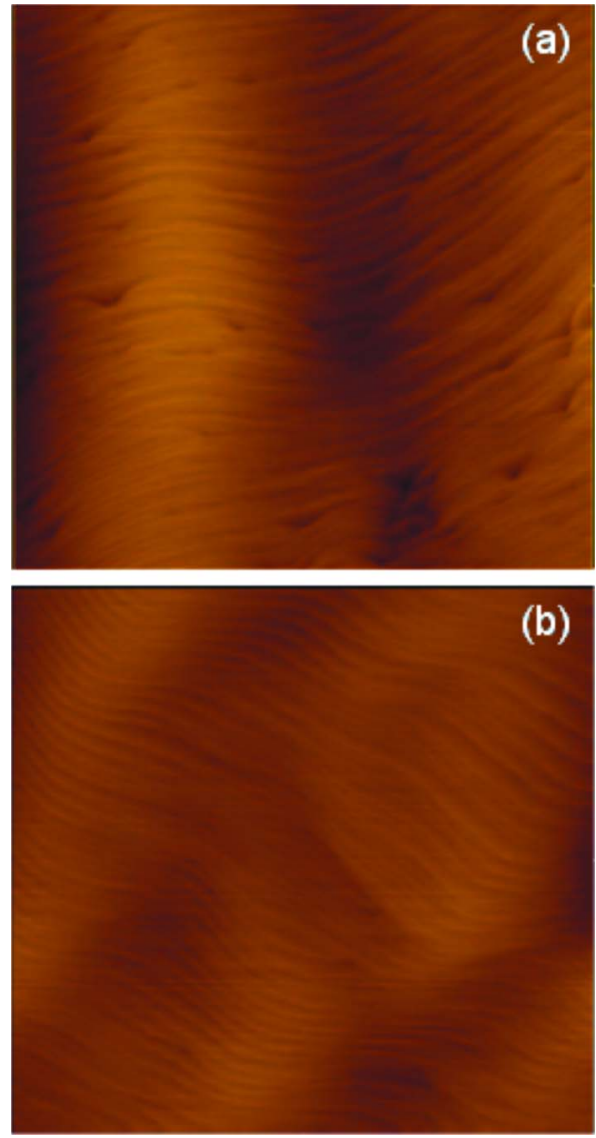

FIG. 3. (Color online) AFM images of $5 \times 5 \mu \mathrm{m}^{2}$ in size of the control sample (a) and the overgrowth sample (b). From the images, the pit density of $3 \times 10^{8} \mathrm{~cm}^{-2}$ and surface roughness of $0.834 \mathrm{~nm}$ in (a) and the pit density of $2 \times 10^{7} \mathrm{~cm}^{-2}$ and surface roughness of $0.411 \mathrm{~nm}$ in (b) can be obtained.

formed at the early stage of coalescence, within the first several hundred nanometer of the coalescence overgrowth. A close look at the SEM image leads to the identification of the domain structure, as shown in Fig. 6(c) (exemplified by the indication of arrows).

Figure 7(a) shows the depth-integrated XRD rocking curves in the (0002) plane of the three samples. In such measurements, the $\mathrm{x}$ ray can penetrate as deep as 5-6 $\mu \mathrm{m}$ in GaN. The FWHMs of the control, nanocolumns, and overgrowth samples are 220, 303, and 256 arc sec, respectively. As shown in Fig. 7(b), the rocking curve of the nanocolumns sample can be decomposed into two components, including the broader one from the nanocolumns and the narrower one from the template beneath. Their FWHMs are 629 and 226 arc sec, respectively. The 226 arc sec of the narrower component is consistent with the 220 arc sec of the control sample. The obtained 303 arc sec of the nanocolumns sample in Fig. 7(a) is mainly contributed by the template layer beneath. The measured 256 arc sec of the overgrowth sample in Fig. 7(a) is slightly overestimated due to the broad rocking curve contribution from the nanocolumns beneath. The broad FWHM (629 arc sec) of the rocking curve in the nanocolumns sample shown in Fig. 7(b) implies that different columns still have significant variations in crystal orientation even though they stem from the same GaN template and each individual nanocolumn can be almost dislocation
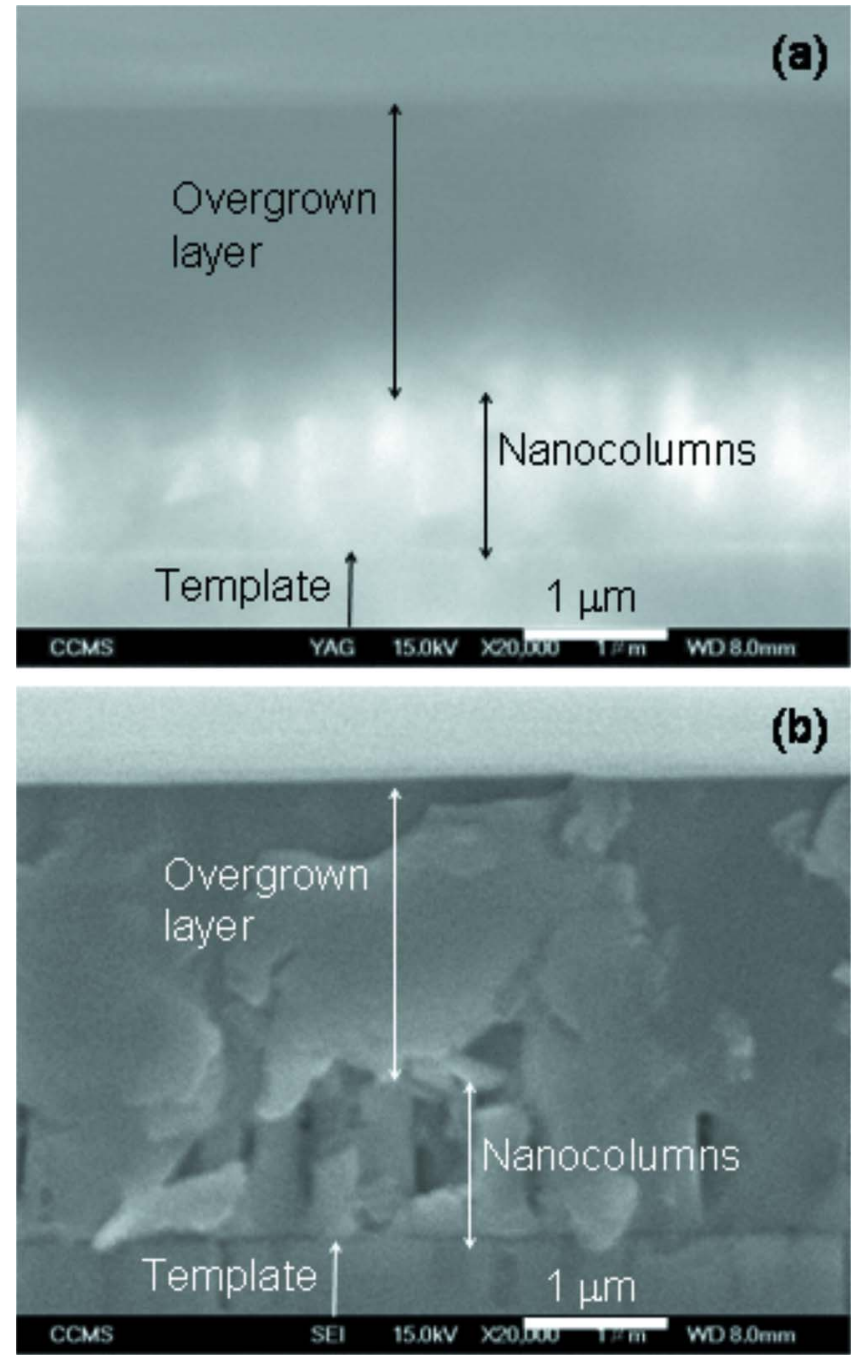

FIG. 4. (Color online) (a) Cross-sectional CL image of the overgrowth sample with an electron acceleration voltage of $15 \mathrm{kV}$. The SEM image taken at the same location as the CL image is shown in part (b). The nanocolumn layer has the higher CL emission efficiency when compared to the other two layers.

free. Because the $\mathrm{GaN}$ template has a coherent domain of a finite size, the growths of the nanocolumns at different locations may start with slightly different crystal orientations. The variations of crystal orientation can be enhanced during the process of column growth because the air gap provides the space for extending the twist and tilt ranges. The crystal orientation variations among various nanocolumns or domains of small size ( $\sim 300 \mathrm{~nm}$ in the lateral dimension) lead to the large FWHM in the XRD rocking curve from the nanocolumns. The XRD data shown in Fig. 7(a) cannot properly reflect the crystal quality of an individual nanocolumn. Meanwhile, they cannot provide us with information about the difference of crystal quality between the overgrown layer and the template layer. Depth-dependent XRD measurement is needed.

Figure 8 shows the depth-dependent rocking curve FWHMs of the control and overgrowth samples based on the three-beam XRD measurement in the $(01-13) /(0-11-2)$ plane. Because of the strong $\mathrm{X}$-ray scattering from the nanocolumn distribution, it becomes difficult to calibrate the depth- 

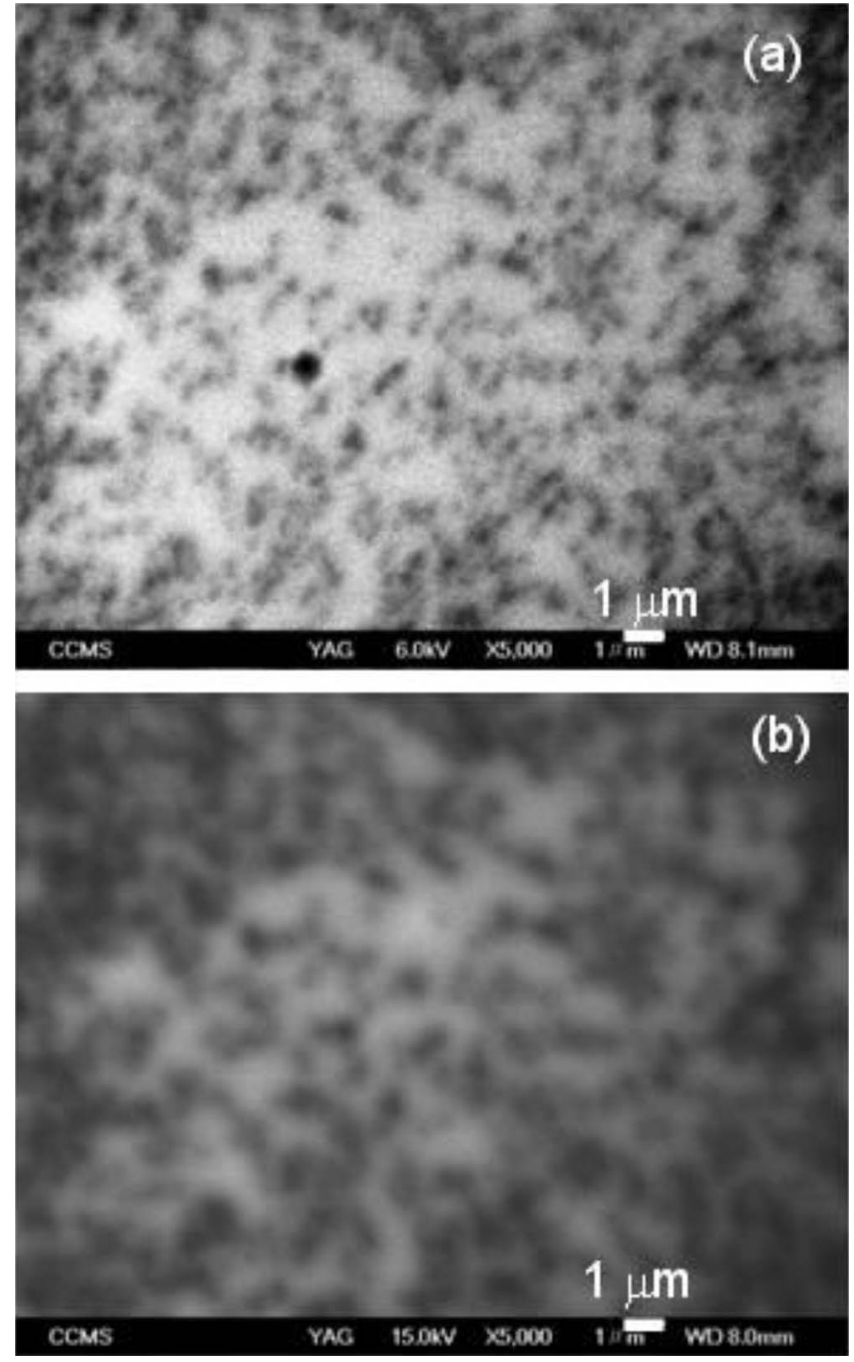

FIG. 5. Plan-view CL images of 5000-time magnification with electron acceleration voltages of $6 \mathrm{kV}$ (a) and $15 \mathrm{kV}$ (b) at about the same location. The larger bright area of stronger emission in a shallower layer (a) can be seen.

dependent crystal quality of nanocolumns. Here, one can see that the crystal quality of the control sample, corresponding to a rocking curve FWHM of $\sim 3400$ arc sec, is quite uniform along depth until the depth beyond $1.6 \mu \mathrm{m}$. In the overgrowth sample, the crystal quality increases along overgrowth thickness until it is saturated at the depth of $\sim 600 \mathrm{~nm}$ to reach $\sim 1700$ arc sec in rocking curve FWHM. In other words, near the top surface of the overgrowth sample, the crystal quality is significantly improved when compared to the $\mathrm{GaN}$ template.

Figure 9 shows the depth-dependent variations of skew dislocation density, edge dislocation density, and lateral domain size of the overgrown layer, which are obtained from the two-beam XRD technique described by Eqs. (1)-(7). One can see that all the three parameter values saturate when the depth is smaller than $600 \mathrm{~nm}$, which is consistent with the result shown in Fig. 8. In other words, under the current nanocolumn growth and coalescence overgrowth conditions, additional overgrowth beyond $\sim 1.5 \mu \mathrm{m}$ in thickness will not further improve the crystal quality. Near the surface of the overgrowth sample, the skew dislocation density, edge
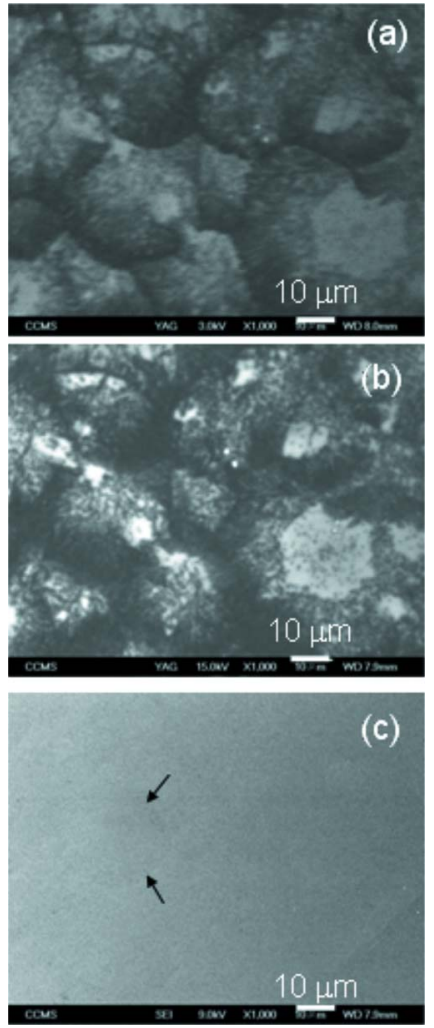

FIG. 6. (Color online) CL images of 1000-time magnification with electron acceleration voltages of $3 \mathrm{kV}$ (a) and $15 \mathrm{kV}$ (b) at about the same location. The domain structure in the overgrown layer with the domain size in the range of several tens of microns can be seen. For reference, the plan-view SEM image of the same location is also shown in part (c). The arrows indicate the boundary of a domain.

dislocation density, and lateral domain size are 2.1 $\times 10^{7} \mathrm{~cm}^{-2}, 4.8 \times 10^{7} \mathrm{~cm}^{-2}$, and $2756 \mathrm{~nm}$, respectively. The crystal quality represented by those values is significantly better than that of the control sample, which is described by the values of $1.1 \times 10^{8} \mathrm{~cm}^{-2}, 6.7 \times 10^{8} \mathrm{~cm}^{-2}$, and $824 \mathrm{~nm}$ for the skew dislocation density, edge dislocation density, and lateral domain size, respectively. The related AFM and XRD measurement results are listed in Table I for clear comparison. Generally, the dislocation density of the overgrowth sample is one order of magnitude smaller than that of the control sample. Also, the lateral domain size near the surface of the overgrowth sample is more than three times larger when compared to that of the control sample. The crystal quality of the overgrowth sample near the surface is significantly better than that of a commonly used GaN thin-film template grown on $c$-plane sapphire substrate, which has threading dislocation density in the range of $10^{9}-10^{10} \mathrm{~cm}^{-2}$. Our control sample represents a particularly good template.

Figure 10 shows the temperature-dependent integrated PL intensities of the three samples. Because the absorption coefficient of $\mathrm{GaN}$ at $325 \mathrm{~nm}$ is around $1 \times 10^{5} \mathrm{~cm}^{-1}$, 22 the PL measurement reflects the optical quality of a layer $\sim 100 \mathrm{~nm}$ in thickness near the surface of the overgrown layer. The ratio of the integrated intensity at room temperature over that at $10 \mathrm{~K}$ is related to the density of nonradiative recombination center. This ratio in the overgrowth sample is about six times that of the control sample (increased from 

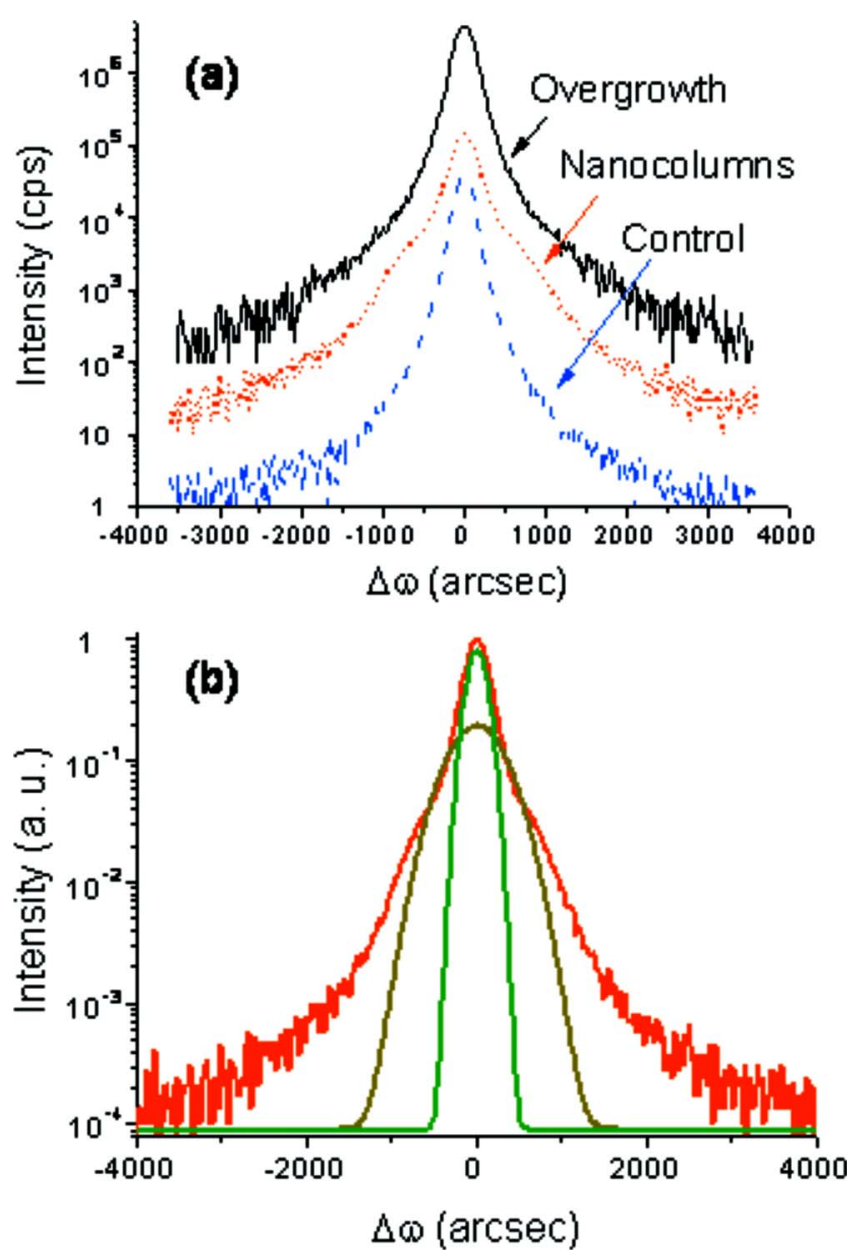

FIG. 7. (Color online) (a) Depth-integrated XRD rocking curves in the (0002) plane of the three samples. The FWHMs of the control, nanocolumns, and overgrowth samples are 220, 303, and 256 arc sec, respectively. As shown in part (b), the rocking curve of the nanocolumns sample can be decomposed into two components, including the broader one from the nanoclumns and the narrower one from the template beneath. Their FWHMs are 629 and 226 arc sec, respectively.

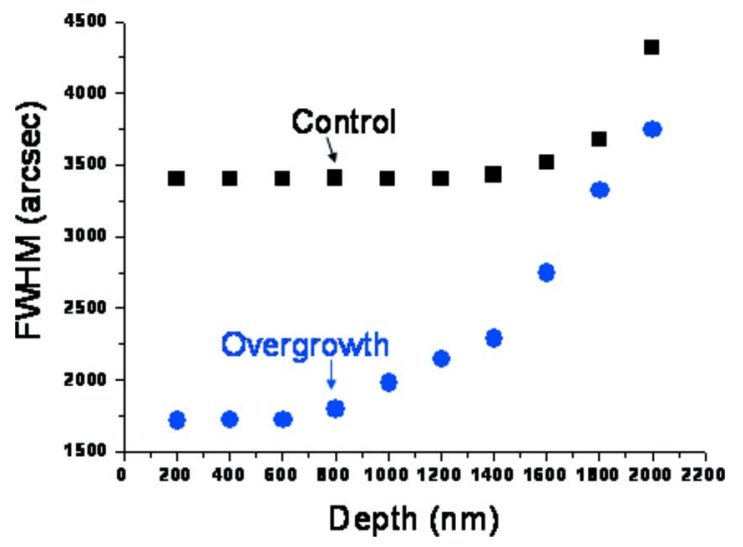

FIG. 8. (Color online) Depth-dependent rocking curve FWHMs of the control and overgrowth samples based on the three-beam XRD measurement in the $(01-13) /(0-11-2)$ plane. Near the top surface of the overgrowth sample, the crystal quality is significantly improved when compared to the GaN template.

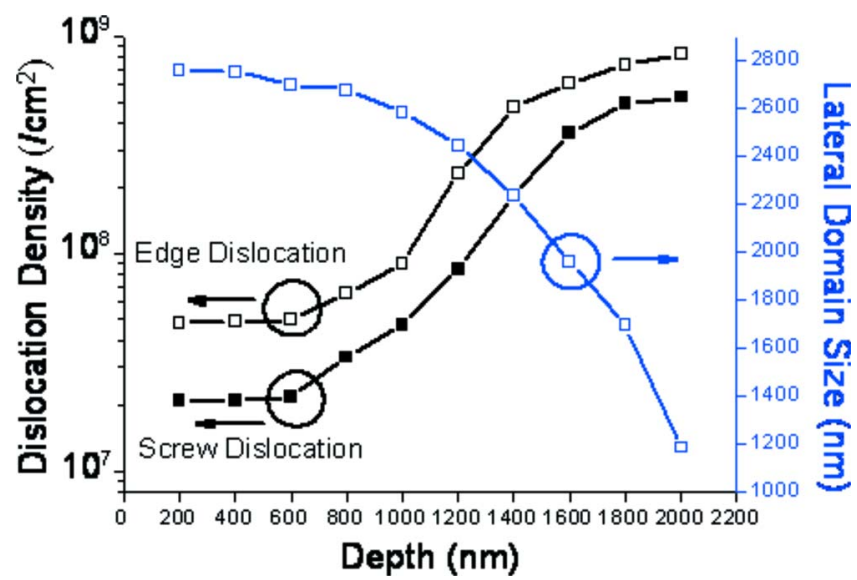

FIG. 9. (Color online) Depth-dependent edge dislocation density, screw dislocation density, and lateral domain size of the overgrowth sample based on the two-beam XRD measurement. The crystal quality represented by those values near the overgrowth surface is significantly better than that of the control sample, which is described by the values of $1.1 \times 10^{8} \mathrm{~cm}^{-2}, 6.7$ $\times 10^{8} \mathrm{~cm}^{-2}$, and $824 \mathrm{~nm}$ for the skew dislocation density, edge dislocation density, and lateral domain size, respectively.

$1.1 \%$ to $6.7 \%$ ), indicating that the density of nonradiative recombination center near the surface of the overgrowth sample is significantly reduced when compared to the control sample. In Fig. 10, the corresponding value of the nanocolumns is also shown as $9.9 \%$, confirming that the optical quality of the nanocolumns is higher than those of the overgrown layer and the control sample.

Figures 11(a) and 11(b) show the normalized PL spectra of the three samples at $10 \mathrm{~K}$ and room temperature, respectively. Here, at either $10 \mathrm{~K}$ or room temperature, one can see that the spectral distributions of the overgrown layer and the control sample are similar. However, that of the nanocolumns sample is significantly redshifted. At $10 \mathrm{~K}$, three peaks in the PL spectra of the overgrowth and control samples, including one major peak around $355.5 \mathrm{~nm}$ due to donorbound exciton recombination and two minor peaks at 354.8 and $356 \mathrm{~nm}$, corresponding to free exciton $\mathrm{A}[\mathrm{FE}(\mathrm{A})]$ and acceptor-bound exciton, respectively. ${ }^{23,24}$ The relatively stronger $\mathrm{FE}(\mathrm{A})$ feature in the overgrowth sample, compared to the control sample, confirms that the defect density in this sample is lower. The PL spectrum of the nanocolumns is quite different. It is redshifted and broadened. The redshift is attributed to the relaxation of compressive strain in the nanocolumns. The spectral broadening mainly results from the

TABLE I. Comparisons of AFM and XRD measurement data between the overgrowth and control samples.

\begin{tabular}{|c|c|c|}
\hline Sample & Control & Overgrowth \\
\hline Surface roughness $(\mathrm{nm})$ & 0.834 & 0.411 \\
\hline Surface pit density $\left(\mathrm{cm}^{-2}\right)$ & $3 \times 10^{8}$ & $2 \times 10^{7}$ \\
\hline $\begin{array}{l}\text { FWHM of the (0002)-plane rocking curve } \\
(\operatorname{arcsec})\end{array}$ & 220 & 256 \\
\hline $\begin{array}{l}\text { FWHM of the }(01-13) /(0-11-2) \text {-plane } \\
\text { rocking curve near surface }(\operatorname{arcsec})\end{array}$ & $\sim 3400$ & $\sim 1700$ \\
\hline Skew dislocation density near surface $\left(\mathrm{cm}^{-2}\right)$ & $1.1 \times 10^{8}$ & $2.1 \times 10^{7}$ \\
\hline Edge dislocation density near surface $\left(\mathrm{cm}^{-2}\right)$ & $6.7 \times 10^{8}$ & $4.8 \times 10^{7}$ \\
\hline Lateral domain size (nm) & 824 & 2756 \\
\hline
\end{tabular}




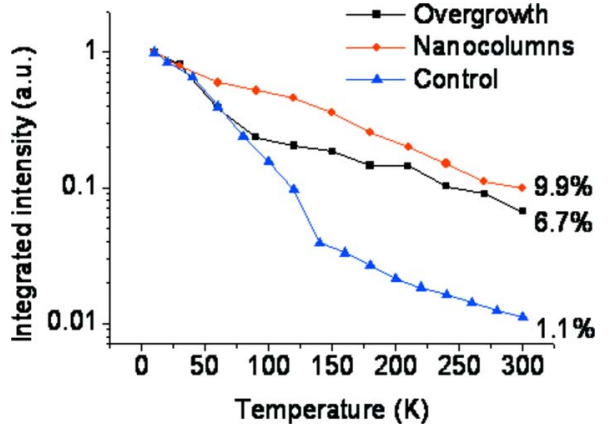

FIG. 10. (Color online) Temperature-dependent integrated PL intensities of the nanocolumns, control, and overgrowth samples. The numbers indicate the ratios of the integrated intensities at $10 \mathrm{~K}$ over those at room temperature for the three samples. Among the three samples, the nanocolumns sample has the highest emission efficiency.

different degrees of strain relaxation among different columns. The single peak of the nanocolumn spectrum (357.1 $\mathrm{nm})$ coincides with the reported value of $\mathrm{FE}(\mathrm{A})$ of fully strain-relaxed GaN (356.8-357.3 nm).$^{1,25,26}$ This coincidence implies that $\mathrm{FE}(\mathrm{A})$ dominates the emission of the nanocolumns. In other words, the densities of donor and acceptor defects are lower in the nanocolumns when compared to the control and overgrowth samples. At room temperature, the two peaks in the PL spectra of all the three samples (around 362 and $368 \mathrm{~nm}$ in the overgrowth and control samples, and around 363.5 and $379 \mathrm{~nm}$ in the nanocolumn sample) are formed because of the existence of a reabsorption feature between the two spectral peaks. ${ }^{27}$ The thermal energy reduces the difference of strain relaxation between the overgrowth (control) and nanocolumns samples such that the separation of the major PL spectral peaks between the two groups of samples is decreased.
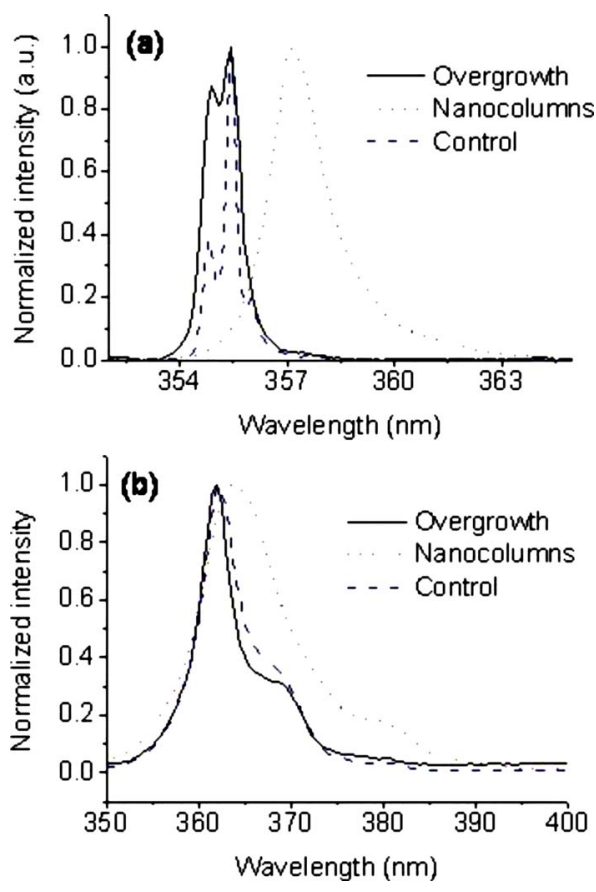

FIG. 11. (Color online) Normalized PL spectra of the three samples at $10 \mathrm{~K}$ (a) and room temperature (b). The PL spectral features of the nanocolumn sample are significantly redshifted from those of the control and overgrowth samples.

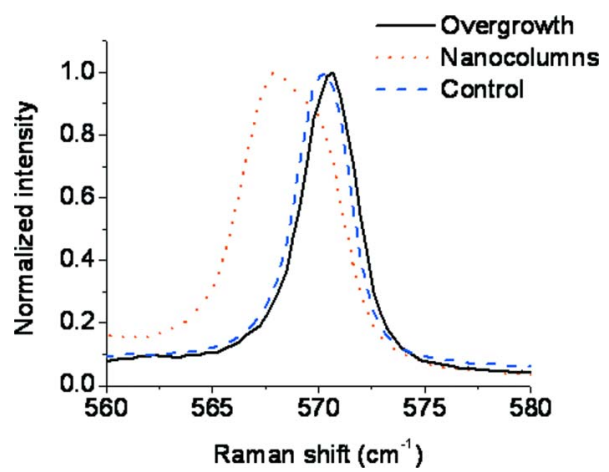

FIG. 12. (Color online) Normalized Raman-shift spectra of the three samples at room temperature. The lower and higher Raman spectral features of the nanocolumn sample correspond to the signals from the nanocolumns and the template beneath, respectively.

Based on the separation of the major spectral peaks between the overgrowth and nanocolumns samples $(1.5 \mathrm{~nm}$ or $14 \mathrm{meV}$ ) in Fig. 11(b), by using the proportionality factor of $K=21.2 \mathrm{meV} / \mathrm{GPa}^{28}{ }^{28}$ we find that at room temperature, a stress of $0.66 \mathrm{GPa}$ is built during the overgrowth process. Because the PL spectral peaks of the control and overgrowth samples are about the same, the stress in the overgrown layer is expected to be about the same as that in the template (the control sample). The confocal micro-Raman spectroscopy is also used as a tool to quantify the stress in GaN according to the frequency position of the $E_{2}$ (high) mode. To improve the depth resolution, the size of the confocal hole was minimized to $30 \mu \mathrm{m}$. The normalized $E_{2}$ (high) mode of the three samples measured at room temperature is shown in Fig. 12. Here, the high Raman-shift shoulder in the curve of the nanocolumn sample corresponds to the signal collected from the template layer beneath. The major peaks of Raman shift in the nanocolumns, overgrowth, and control samples are $567.9,570.7,570.4 \mathrm{~cm}^{-1}$, respectively. Therefore, the difference in Raman shift between the overgrowth layer and the nanocolumns is $2.8 \mathrm{~cm}^{-1}$. Based on the stress coefficient of $4.2 \mathrm{~cm}^{-1} \mathrm{GPa}^{-1}{ }^{29}$ one can estimate the stress built in the overgrown layer to give $0.67 \mathrm{GPa}$, which is quite consistent with that estimated from the PL spectral shift.

\section{CONCLUSIONS}

In summary, we have demonstrated the high-quality coalescence overgrowth of patterned-grown $\mathrm{GaN}$ nanocolumns on $c$-plane sapphire substrate with MOCVD. Although domain structures of a tens of micron scale in the overgrown layer were identified with CL measurement, the surface roughness of the overgrown layer in an area of $5 \times 5 \mu \mathrm{m}^{2}$ was only $0.411 \mathrm{~nm}$, which was about one-half that of the $\mathrm{GaN}$ template for nanocolumn growth (the control sample). Based on the AFM and depth-dependent XRD measurements, near the surface of the overgrown layer, the dislocation density was reduced to the order of $10^{7} \mathrm{~cm}^{-2}$, which is one order of magnitude lower than that of the control sample and two to three orders of magnitude lower than those of ordinary GaN templates for LED fabrication. Also, the lateral domain size was increased to a level of $\sim 2.7 \mu \mathrm{m}$, which is more than three times that of the control sample. 
The ratio of PL intensity at room temperature over that at a low temperature of the overgrown sample was at least six times higher than that of the control sample. Although the strain in nanocolumns was almost completely released, a stress of $\sim 0.66 \mathrm{GPa}$ was rebuilt when the coalescence overgrowth was implemented.

\section{ACKNOWLEDGMENTS}

This research was supported by the National Science Council, The Republic of China, under Grant Nos. NSC 962120-M-002-008 and NSC 96-2628-E-002-044-MY3, by the Excellent Research Projects of National Taiwan University under Grant No. 97R0061-04, and by the U.S. Air Force Scientific Research Office under Contract No. AOARD-074010 .

${ }^{1}$ J. E. Van Nostrand, K. L. Averett, R. Cortez, J. Boeckl, C. E. Stutz, N. A. Sanford, A. V. Davydov, and J. D. Albrecht, J. Cryst. Growth 287, 500 (2006).

${ }^{2}$ K. Kouyama, M. Inoue, Y. Inose, N. Suzuki, H. Sekiguchi, H. Kunugita, K. Ema, A. Kikuchi, and K. Kishino, J. Lumin. 128, 969 (2008).

${ }^{3}$ L. Cerutti, J. Ristiæ, S. Fernández-Garrido, E. Calleja, A. Trampert, K. H. Ploog, S. Lazic, and J. M. Calleja, Appl. Phys. Lett. 88, 213114 (2006).

${ }^{4}$ Y. Inoue, T. Hoshino, S. Takeda, K. Ishino, A. Ishida, H. Fujiyasu, H. Kominami, H. Mimura, Y. Nakanishi, and S. Sakakibara, Appl. Phys. Lett. 85, 2340 (2004).

${ }^{5}$ Y. S. Park, C. M. Park, D. J. Fu, T. W. Kang, and J. E. Oh, Appl. Phys. Lett. 85, 5718 (2004).

${ }^{6}$ A. Kikuchi, M. Kawai, M. Tada, and K. Kishino, Jpn. J. Appl. Phys., Part 2 43, L1524 (2004).

${ }^{7}$ M. Yoshizawa, A. Kikuchi, M. Mori, N. Fujita, and K. Kishino, Jpn. J. Appl. Phys., Part 2 36, L459 (1997).

${ }^{8}$ E. Calleja, M. A. Sanchez-Garcia, F. J. Sanchez, F. Calle, F. B. Naranjo, E. Munoz, S. I. Molina, A. M. Sanchez, F. J. Pacheco, and R. Garcia, J. Cryst. Growth 201-202, 296 (1999).

${ }^{9}$ X. Wang, X. Sun, M. Fairchild, and S. D. Hersee, Appl. Phys. Lett. 89, 233115 (2006)
${ }^{10}$ T. Kuykendall, P. J. Pauzauskie, Y. Zhang, J. Goldberger, D. Sirbuly, J. Denlinger, and P. Yang, Nature Mater. 3, 524 (2004).

${ }^{11}$ B. Y. Shim, E. A. Ko, J. C. Song, D. H. Kang, D. W. Kim, I. H. Lee, S. Kannappan, and C. R. Lee, Jpn. J. Appl. Phys., Phys. 1 46, 2571 (2007).

${ }^{12}$ S. D. Hersee, X. Sun, and X. Wang, Nano Lett. 6, 1808 (2006).

${ }^{13}$ K. Kusakabe, A. Kikuchi, and K. Kishino, Jpn. J. Appl. Phys., Part 2 40, L192 (2001).

${ }^{14}$ T. Y. Tang, K. L. Averett, J. D. Albrecht, W. Y. Shiao, Y. S. Chen, C. C. Yang, C. W. Hsu, and L. C. Chen, Nanotechnology 18, 445601 (2007).

${ }^{15}$ W. Y. Shiao, T. Y. Tang, Y. S. Chen, K. L. Averett, J. D. Albrecht, and C. C. Yang, J. Cryst. Growth 310, 3159 (2008).

${ }^{16}$ S. L. Morelhao and L. P. Cardoso, J. Appl. Phys. 73, 4218 (1993).

${ }^{17}$ S. L. Morelhao and E. Abramof, J. Appl. Crystallogr. 32, 871 (1999).

${ }^{18}$ A. Reiher, J. Blasing, A. Dadgar, and A. Krost, Appl. Phys. Lett. 84, 3537 (2004).

${ }^{19}$ S. R. Lee, A. M. West, A. A. Allerman, K. E. Waldrip, D. M. Follstaedt, P. P. Provencio, and D. D. Koleske, Appl. Phys. Lett. 86, 241904 (2005).

${ }^{20}$ K. Y. Zang, Y. D. Wang, H. F. Liu, and S. J. Chua, Appl. Phys. Lett. 89, 171921 (2006).

${ }^{21}$ S. W. Feng, T. Y. Tang, Y. C. Lu, S. J. Liu, E. C. Lin, C. C. Yang, K. J. Ma, C. H. Shen, L. C. Chen, J. Y. Lin, and H. X. Jiang, J. Appl. Phys. 95, 5388 (2004).

${ }^{22}$ J. F. Muth, J. H. Lee, I. K. Shmagin, R. M. Kolbas, H. C. Casey, Jr., B. P. Keller, U. K. Mishra, and S. P. DenBaars, Appl. Phys. Lett. 71, 2572 (1997).

${ }^{23}$ B. Monemar, Mater. Sci. Eng., B 59, 122 (1999).

${ }^{24}$ M. G. Tkachman, T. V. Shubina, V. N. Jmerik, S. V. Ivanov, P. S. Kopev, T. Paskova, and B. Monemar, Semiconductors 37, 532 (2003).

${ }^{25}$ Y. D. Wang, S. J. Chua, S. Tripathy, M. S. Sander, P. Chen, and C. G. Fonstad, Appl. Phys. Lett. 86, 071917 (2005).

${ }^{26}$ B. Monemar, J. P. Bergman, I. A. Buyanova, H. Amano, I. Akasaki, T. Detchprohm, K. Hiramatsu, and N. Sawaki, Solid-State Electron. 41, 239 (1997).

${ }^{27}$ G. E. Bunea, W. D. Herzog, M. S. Unlu, B. B. Goldberg, and R. J. Molnar, Appl. Phys. Lett. 75, 838 (1999).

${ }^{28}$ D. G. Zhao, S. J. Xu, M. H. Xie, S. Y. Tong, and H. Yang, Appl. Phys. Lett. 83, 677 (2003).

${ }^{29}$ C. Kisielowski, J. Kruger, S. Ruvimov, T. Suski, J. W. Ager III, E. Jones, Z. Liliental-Weber, M. Rubin, E. R. Weber, M. D. Bremser, and R. F. Davis, Phys. Rev. B 54, 17745 (1996). 